\title{
Associations of Subjective Memory Complaints and Simple Memory Task Scores With Future Dementia in the Primary Care Setting
}

Lennard L. van Wanrooij, MSc ${ }^{1}$

Edo Richard, $M D, P b D^{2}$

Susan Jongstra, $M D, P b D^{1}$

Eric P. Moll van Charante, MD,

$\mathrm{PbD}^{3}$

Willem A. van Gool, MD

${ }^{1}$ Amsterdam UMC, University of

Amsterdam, Department of Neurology,

Amsterdam, The Netherlands

${ }^{2}$ Donders Institute of Brain, Cognition, and Behavior, Department of Neurology, Radboud University Medical Center, Nijmegen, The Netherlands

${ }^{3}$ Amsterdam UMC, University of Amsterdam, Department of Family Medicine, Amsterdam Public Health Research Institute, Amsterdam, The Netherlands

Conflicts of interest: authors report none.

\section{CORRESPONDING AUTHOR}

Lennard L. van Wanrooij, MSc

Amsterdam UMC

University of Amsterdam, Department of

Neurology

Meibergdreef 9, $1105 \mathrm{AZ}$

Amsterdam, The Netherlands

1.1.vanwanrooij@amc.uva.nl

\begin{abstract}
PURPOSE Family physicians need simple yet comprehensive algorithms to discriminate between community-dwelling older persons who are at increased risk of dementia and those who are not. We aimed to investigate associations between incident dementia and responses to a single question regarding subjective memory complaints (SMC) combined with scores on 2 simple memory tests that are easy to use in the primary care setting.
\end{abstract}

METHODS Analyses were based on data from 3,454 community-dwelling older persons who participated in the 6- to 8-year Prevention of Dementia by Intensive Vascular Care (preDIVA) trial, yielding 21,341 person-years of observation. Participants were considered a single cohort. We used Cox models to assess separate and combined associations of SMC, an imperfect score on the Mini-Mental State Examination delayed recall item (MMSE-5), and an imperfect score on the Visual Association Test (VAT) with future dementia.

RESULTS Subjective memory complaints alone were associated with future dementia (hazard ratio $[\mathrm{HR}]=3.01 ; 95 \% \mathrm{Cl}, 2.31-3.94 ; P<.001$ ), as were the MMSE-5 (HR $=2.14 ; 95 \% \mathrm{Cl}, 1.59-2.87 ; P<.001)$ and VAT $(\mathrm{HR}=3.19 ; 95 \% \mathrm{Cl}$, $2.46-4.13 ; P<.001)$ scores. After a median follow-up of 6.7 years, the occurrence of dementia ranged from $4 \%$ to $30 \%$ among persons with SMC, depending on the MMSE- 5 and VAT scores. These test scores did not substantially alter the association with future dementia for persons without SMC.

CONCLUSIONS In persons with SMC, the strength of the association between future dementia and an imperfect MMSE-5 score depends substantially on the VAT score.

Ann Fam Med 2019;17:412-418. https://doi.org/10.1370/afm.2443.

\section{INTRODUCTION}

A timely dementia diagnosis provides both patients and caregivers the opportunity to adapt to functional deterioration and to initiate appropriate care and treatment. ${ }^{1}$ Therefore, it is important that family physicians (FPs) be able to identify community-dwelling older persons at increased risk of dementia. Embedding a screening question regarding subjective memory complaints (SMC) in general primary care might be a useful first step in this process, given that a recent review showed that SMC, even without objective cognitive impairment, is associated with future dementia. ${ }^{2}$ That review included studies with different measures for SMC, including studies that used a single question to assess SMC, but did not report to what extent associations of SMC with future dementia are dependent on scores on memory tests. It could be helpful for FPs to differentiate among older persons with SMC between those with or without a substantially increased risk of dementia. Monitoring or referral to specialist memory clinics might be warranted for some older persons with SMC, but for others this would cause unnecessary stress, and this would place too much of a burden on health care resources. A single question regarding SMC 
combined with a simple and brief memory assessment could fit within a regular visit to an FP.

In primary care settings, the Mini-Mental State Examination (MMSE) is often used as a cognitive screening instrument. Unfortunately, most studies on the association between MMSE score and dementia have been cross-sectional. Longitudinal studies on this topic are lacking. Nonetheless, on the basis of previous (cross-sectional) studies, several recommendations can be made to FPs with regard to cognitive assessments that are suitable for use in primary care. For example, it could be useful to focus on the score of the MMSE delayed recall item (item 5 [MMSE-5]) to discriminate between older persons with and without increased dementia risk, rather than on the MMSE sum score, given that this item primarily drives the association between the MMSE sum score and cognitive decline or onset of dementia. ${ }^{3-5}$ Whereas the MMSE is often used in primary care, a cross-sectional study showed that the Visual Association Test (VAT) is superior to several other cognitive tasks often used in primary care with regard to the recognition of dementia. ${ }^{6}$ The VAT is a language-independent test of anterograde memory assessment, which is easy to use and takes only a few minutes to administer. ${ }^{7,8}$ Recently, we showed that the VAT helps to distinguish among older persons, whose MMSE sum score decreased over the past 2 years, those with and without elevated dementia risk. ${ }^{9}$

However, it is unknown to what extent a combination of MMSE-5 and VAT scores is associated with future dementia in situations in which these tests are administered during a single memory assessment, and whether this differs in older persons with SMC. Therefore, we analyzed long-term individual and combined associations of SMC assessed via a single question, the MMSE-5, and the VAT with future dementia among community-dwelling older persons.

\section{METHODS}

\section{Participants}

Individual participant data were derived from the Prevention of Dementia by Intensive Vascular Care (preDIVA) trial. ${ }^{10}$ In brief, this cluster-randomized controlled trial tested the efficacy of a nurse-led, multicomponent, cardiovascular intervention to prevent all-cause dementia among 3,526 community-dwelling elderly persons aged 70 to 78 years at baseline, of which $98 \%$ were white. An exclusion criterion for the preDIVA trial was a (suspected) dementia diagnosis at enrollment. Eligible individuals were recruited from 2006 to 2009, and median follow-up was 6.7 years. Participants in the intervention group visited a study nurse every 4 months, who addressed all cardiovas- cular risk factors. The control population received standard care. For the present study, the population was considered a single cohort. We deemed this appropriate given that the overall results of the main trial were neutral, although we conducted several sensitivity analyses to explore the potential effect of adding randomization group as a covariate. At baseline and at 2-year intervals, we collected data on medical history, medication use, cardiovascular risk factors, cognitive functioning, and mood. The details of the preDIVA trial have been published. ${ }^{10,11}$

\section{Assessment}

\section{Dementia Diagnosis}

Dementia diagnosis was assessed at 2-year intervals and supplemented by electronic health record data. These records were accessed via FPs and included reports on hospital admissions; outpatient diagnostic evaluations by geriatricians, neurologists, and psychiatrists; and neuroimaging and/or neuropsychologic examinations. Diagnosis of dementia was based on Diagnostic and Statistical Manual of Mental Disorders, 4th edition, ${ }^{12}$ criteria. An independent outcome adjudication committee consisting of neurologists, old-age psychiatrists, geriatricians, cardiologists, and FPs meticulously evaluated all dementia diagnoses and was blinded to any additional information from the study. As a quality check and to minimize the risk of false-positive diagnoses, dementia diagnoses were re-evaluated on the basis of the clinical course after 1 year. Diagnostic procedures and outcome adjudication have been described in detail in the supplementary appendix to the primary publication for the preDIVA trial. ${ }^{10}$

\section{Subjective Memory Complaints}

To assess SMC, we used a dichotomous question of the 15-item Geriatric Depression Scale administered at baseline and each 2-year follow-up (item 10), "Do you feel you have more problems with memory than most?"13

\section{Mini-Mental State Examination}

We used the MMSE-5 delayed recall item, in which the Dutch translations for the terms apple, key, and table were to be recalled after a short delay, with a score range of 0 to $3 .{ }^{14,15}$ The MMSE- 5 score was converted to a dichotomous score of optimal (3 points) or imperfect (0-2 points).

\section{Visual Association Test}

The VAT is a test of visual anterograde memory. ${ }^{7,8} \mathrm{We}$ used version A of the short form, which consists of 6 images of 2 semantically unrelated, interacting objects (eg, a balloon and a key). The images are shown one after another, and participants are asked to name both 
objects. After a short delay, only the cue objects (eg, the balloon) are shown. The VAT score is equal to the number of target objects (eg, the key) participants are able to recall (range, 0-6). The VAT scores were also transformed to dichotomous scores of optimal (6 points) or imperfect (0-5 points). We applied this strict cut-off because a (presumably) cognitively intact population was under study. ${ }^{7}$

\section{Assessor}

The 15 -item Geriatric Depression Scale, MMSE, and VAT, along with other measures assessed for the parent trial, were administered by trained nurses associated with the study.

\section{Statistical Analyses}

For all analyses, we used Cox proportional hazards regression models. Time-to-event was calculated by subtracting the days from the date of the preDIVA baseline visit to the date of dementia diagnosis. Participants who died during the study without dementia and those free of dementia at the end of the trial were censored on the day they left the study. The assumption of proportional hazards was checked for each model.

We first fit 3 models with incident dementia at follow-up as the dependent variable and SMC, MMSE-5 score, or VAT score at baseline as the predictor to assess the individual predictive value of these memory measurements for incident dementia. We subsequently created a categoric variable that contained 4 levels, in which SMC was combined with MMSE-5 score as follows: (1) no SMC and optimal MMSE-5, (2) no SMC and imperfect MMSE-5, (3) SMC and optimal MMSE-5, and (4) SMC and imperfect MMSE-5. This was performed to assess the incremental value of the MMSE-5 score and the presence or absence of SMC for an association with dementia. We also created such a variable for the combination of SMC with the dichotomous VAT score and a variable that contained 8 levels, in which SMC was combined with both the MMSE-5 score and VAT score. We performed several sensitivity analyses using less strict cut-offs for MMSE-5 and VAT (1 and 4 correct items, respectively). In addition, analyses were repeated with the MMSE sum score replacing the MMSE-5 score. We adjusted for age and educational level in all models. We used R Studio, ${ }^{16}$ specifically the packages surviva ${ }^{17}$ and ggsurvplot ${ }^{18}$ for all analyses. To summarize our main results we created a tree graph of 4 levels, starting with the overall dementia occurrence in our sample. Layers for SMC, MMSE-5, and
VAT were added in that order. Confidence intervals were calculated by subtracting and adding the standard deviation of participants who developed dementia per group, multiplied by 1.96 , which is the $97.5 \%$ percentile approximation of a normal distribution.

\section{RESULTS}

Analyses were conducted for 3,454 of 3,526 preDIVA participants $(98 \%)_{i} 72$ (2\%) participants for whom data on dementia diagnosis at follow-up were missing were excluded. ${ }^{10}$ This group $(\mathrm{n}=72)$ did not differ with respect to age, sex, education, baseline MMSE score, VAT score, or response to the SMC question compared to the group included for analysis (Supplemental Table 1, http://www.AnnFamMed.org/content/17/5410/suppl/ DC1). After a median of 6.7 years of follow-up, 233 of the 3,454 participants (7\%) had developed dementia (median dementia incidence: 60 months $_{i}$ interquartile range: 39-74 months). The baseline characteristics of the study sample are summarized in Table 1.

Dementia occurred in $146 / 2,822$ (5\%) of participants without SMC at baseline and in $86 / 601$ (14\%) of participants with SMC (hazard ratio $[\mathrm{HR}]=3.01 ; 95 \%$ CI, 2.31-3.94; $P<.001$ ) (Table 2). Whereas dementia developed in 59/1,403 (4\%) of participants with an optimal MMSE-5 score at baseline, it occurred in $174 / 2,050(8 \%)$ of participants with an imperfect baseline score $(\mathrm{HR}=2.14 ; 95 \% \mathrm{CI}, 1.59-2.87 ; \mathrm{P}<.001)$. With regard to the VAT, dementia was diagnosed for $106 / 2454(4 \%)$ of participants with an optimal baseline score compared to $124 / 982(13 \%)$ with an imperfect baseline score $(\mathrm{HR}=3.19 ; 95 \% \mathrm{CI}, 2.46-4.13 ; \mathrm{P}<.001)$.

Table 1. Baseline Patient Characteristics

\begin{tabular}{|c|c|c|}
\hline Characteristic & $\begin{array}{l}\text { No Dementia } \\
\text { at Follow-up } \\
(\mathrm{n}=3,221)\end{array}$ & $\begin{array}{l}\text { Dementia } \\
\text { at Follow-up } \\
(n=233)\end{array}$ \\
\hline Mean age, y (SD; IQR) & $74.3(2.5 ; 72-76)$ & $75.1(2.6 ; 73-77)$ \\
\hline Female, No. (\%) & $1,752(54)$ & $133(57)$ \\
\hline \multicolumn{3}{|l|}{ Educational level, No. (\%) ${ }^{\mathrm{a}}$} \\
\hline Low: $<7$ y & $748(23)$ & $74(32)$ \\
\hline Intermediate: $7-12$ y & $2,007(63)$ & $133(58)$ \\
\hline High: $>12$ y & $436(14)$ & $22(10)$ \\
\hline Subjective memory complaints, No. $(\%)^{b}$ & $515(16)$ & $86(37)$ \\
\hline MMSE-5 imperfect, No. (\%)c & $1,876(58)$ & $174(75)$ \\
\hline VAT imperfect, No. (\%) & $858(27)$ & $124(54)$ \\
\hline \multicolumn{3}{|c|}{$\begin{array}{l}\text { IQR = interquartile range; MMSE-5 = Mini-Mental State Examination, item } 5 \text { (delayed recall); } \\
\text { VAT = Visual Association Test. }\end{array}$} \\
\hline \multicolumn{3}{|c|}{ a 34 missing (30 in no dementia group, 4 in dementia group). } \\
\hline \multicolumn{3}{|c|}{$\begin{array}{l}\text { b } 31 \text { missing, based on Geriatric Depression Scale 15, item } 10 \text { (30 in no dementia group, } 1 \text { in } \\
\text { dementia group). }\end{array}$} \\
\hline \multicolumn{3}{|c|}{ ' 1 missing (in no dementia group). } \\
\hline d 18 missing ( 15 in no dementia group, 3 in d & & \\
\hline
\end{tabular}


Table 2. Dementia Risk in Relation to SMC and Scores on the MMSE-5 and VAT

\begin{tabular}{|c|c|c|c|c|c|}
\hline Variable & $\begin{array}{c}\text { No. Dementia CasesI } \\
\text { No. at Risk (\%) }\end{array}$ & HR $(95 \% \mathrm{CI})$ & $P$ Value & $\begin{array}{l}\text { Adjusted HR } \\
(95 \% \mathrm{Cl})^{\mathrm{a}}\end{array}$ & $P$ Value \\
\hline No SMC & $146 / 2,822(5)$ & 1 (reference) & & & \\
\hline SMC & 86/601 (14) & $3.01(2.31-3.94)$ & $<.001$ & $2.97(2.27-3.9)$ & $<.001$ \\
\hline Optimal MMSE-5 (3 points) & $59 / 1,403(4)$ & 1 (reference) & & & \\
\hline Imperfect MMSE-5 (<3 points) & $174 / 2,050(8)$ & $2.14(1.59-2.87)$ & $<.001$ & $1.98(1.47-2.67)$ & .007 \\
\hline Imperfect VAT (<6 points) & $124 / 982(13)$ & $3.19(2.46-4.13)$ & $<.001$ & $3.04(2.33-3.95)$ & $<.001$ \\
\hline
\end{tabular}

Table 3. Dementia Risk in Relation to SMC in Combination With Either the MMSE-5 or VAT

\begin{tabular}{|c|c|c|c|c|c|}
\hline Variable & $\begin{array}{c}\text { No. of Dementia } \\
\text { Cases/No. at Risk (\%) }\end{array}$ & HR (95\% CI) & $P$ Value & $\begin{array}{l}\text { Adjusted HR } \\
\qquad(95 \% \mathrm{Cl})^{\mathrm{a}}\end{array}$ & $P$ Value \\
\hline No SMC and optimal MMSE-5 $5^{\text {b }}$ & $48 / 1,176(4)$ & 1 (reference) & & & \\
\hline No SMC and imperfect MMSE-5 & $98 / 1,645(6)$ & $1.52(1.08-2.15)$ & .017 & $1.41(1.0-2.0)$ & .056 \\
\hline SMC and optimal MMSE-5 & $11 / 210(5)$ & $1.33(0.69-2.56)$ & .394 & $1.32(0.69-2.55)$ & .401 \\
\hline SMC and imperfect MMSE-5 & 75/391 (19) & $5.48(3.82-7.88)$ & $<.001$ & $5.06(3.5-7.3)$ & $<.001$ \\
\hline No SMC and optimal VATc & $77 / 2,060(4)$ & 1 (reference) & & & \\
\hline No SMC and imperfect VAT & $68 / 749(9)$ & $2.62(1.89-3.63)$ & $<.001$ & $2.56(1.84-3.57)$ & $<.001$ \\
\hline SMC and optimal VAT & 29/373 (8) & $2.25(1.45-3.45)$ & $<.001$ & $2.33(1.52-3.58)$ & $<.001$ \\
\hline SMC and imperfect VAT & $55 / 223(25)$ & $7.74(5.47-10.94)$ & $<.001$ & $7.15(5.01-10.19)$ & $<.001$ \\
\hline
\end{tabular}

The incidence of dementia was $4 \%$ for participants free of SMC who reached an optimal score on either the MMSE-5 or the VAT (Table 3). Incident dementia rates were similar for participants without SMC who failed to reach a perfect score on the MMSE-5 (6\%) or VAT (9\%). Incident dementia rates were greatest for participants with SMC who had an imperfect score on the MMSE-5 (19\%) or the VAT (25\%). We also examined incident dementia rates for all combinations of presence or absence of SMC with perfect or imperfect scores on the MMSE-5 and VAT. Hazard ratios for these analyses are shown in Supplemental Table 2 (http://www.AnnFamMed.org/ content/17/5/412/suppl/DC1).

Dementia rates per group are shown in Figure 1, starting with the a priori dementia risk in our study population of $7 \%$. The time to diagnosis is shown in Figure 2. Of participants without SMC at baseline, 5\% developed dementia, whereas adding the MMSE-5 and VAT scores made this incidence vary between 3\% and 9\% (Figure 1). With regard to the $14 \%$ of participants with SMC at baseline who developed dementia, adding the MMSE-5 and VAT scores substantially changed the percentages of future dementia cases per test score group dementia occurred in $4 \%$ of those with optimal scores on the 2 tests and in $30 \%$ of those with imperfect scores on the 2 tests. Supplemental Table 3 (http://www.AnnFamMed.org/content/17/5/412/suppl/ DC1) was added to show why we included age and education as covariates in our models but not sex. Supplemental Table 4 (http://www.AnnFamMed.org/ content/17/5/412/suppl/DC1) highlights the point that randomization in the parent trial did not influence the results of the present study. In Supplemental Tables 5, 6, and 7 (http://www.AnnFamMed.org/ content/17/5/412/suppl/DC1), the results of sensitivity analyses using less strict cut-offs for MMSE-5 and VAT scores are shown, and in Supplemental Tables 8 and 9 (http://www.AnnFamMed.org/content/17/5/412/ suppl/DC1), analyses were added using MMSE sum scores instead of MMSE-5 scores. Applying less strict test cut-off scores appeared to lead to greater specificity for dementia (17/39 [44\%] older persons with a combination of SMC, MMSE- $5<2$, and VAT $<5$ developed dementia), but given the small group sizes, we considered these results to be less reliable. Replacing MMSE- 5 scores with MMSE sum scores did not alter the results substantially. 


\section{Figure 1. Tree graph of incident dementia rates for SMC in combination with MMSE delayed recall item} scores and VAT scores.

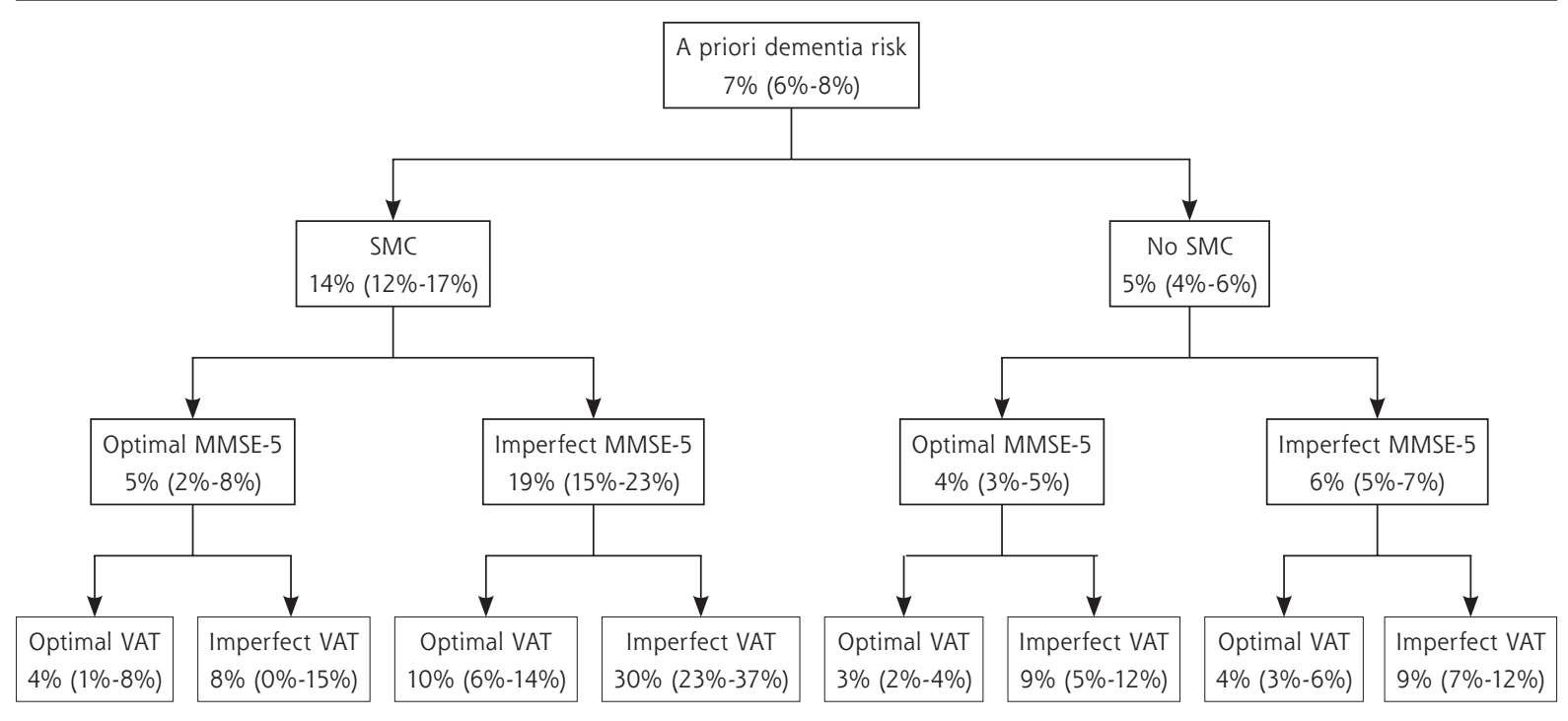

MMSE = Mini-Mental State Examination, item 5 (delayed recall); SMC = subjective memory complaints; VAT = Visual Association Test.

Note: Data are presented as mean $(95 \% \mathrm{Cl})$.

For the main and sensitivity analyses together, we used a total of 51 Cox models. Because most predictors were categoric variables with $>2$ levels, the Cox proportional hazards assumption was met for 166 of 186 predictors (89\%). There was 1 predictor in the main analyses for which the assumption was not met, namely the model in which SMC was combined with imperfect VAT score.

\section{DISCUSSION}

Our results show that a single question regarding memory complaints in community-dwelling older persons is comparably associated with the development of dementia as the score on the MMSE delayed recall item, the MMSE sum score, or a specific test of anterograde visual memory (VAT). For older persons with SMC, subsequently administering the MMSE and VAT substantially changed the percentages of future dementia cases, yielding $4 \%$ and $30 \%$ in those with 2 optimal and imperfect scores, respectively. In older persons without SMC, further cognitive testing or monitoring does not appear to be warranted, given that it did not lead to substantial changes in the association with future dementia. In situations in which an FP has the opportunity to administer only 1 test, perhaps because of time constraints, for an older person with SMC, the VAT appears to be a little more useful than the MMSE-5. However, the VAT appears to be most useful for older persons reporting SMC who do not reach an optimal score on the MMSE-5. Therefore, we suggest the following stepwise approach for primary care settings: first ask a question regarding SMC, then administer the MMSE-5 to those with SMC, and as a last step, administer the VAT to those who fail to remember all 3 MMSE-5 objects.

\section{Strengths and Limitations}

Strengths of the present study include the large sample size of community-dwelling older persons, the high retrieval percentage with regard to dementia outcome, and the robust dementia assessment throughout the full 6 to 8 years of follow-up of the trial. With respect to limitations, first, a small proportion of the Cox analyses failed to meet the assumption of proportional hazards. However, this mainly occurred for sensitivity analyses, for which the results were similar to those for the main analyses. The only main analysis in which this occurred was for the variable in which the presence of SMC was combined with an imperfect score on the VAT, which might indicate that this specific scoring profile is associated more with dementia over the short-term rather than the long-term. A second limitation was the use of an item from a depression questionnaire as a measure for SMC. Contradictory to a report by Mitchell et $\mathrm{al}_{1}{ }^{2}$ we found that SMC was not associated with future dementia in the absence of objective cognitive decline on tests. It is possible that for some participants, an indicative response to item 10 on the 15 -item Geriatric Depression Scale might have 
been an expression of depressed mood rather than of true SMC, which might have caused this contradiction. Therefore, conceptual replication of the present study is desired using a specific question on memory problems, although item 10 on the 15 -item Geriatric Depression Scale has been used as an instrument for SMC. ${ }^{19}$ It nonetheless appears promising to be able to assess SMC via a single question, given the time constraints in primary care settings. Third, although the VAT is easy and quick to administer, it currently appears to be copyrighted and not yet available for broad application. Fourth, the present study is not fully generalizable as performed, given that the age range of preDIVA participants was somewhat narrow (70-78 years), and those for whom long-term follow-up was not considered feasible were excluded at baseline. This might have led to the exclusion of frail older persons, including those with (impending) cognitive impair- ment, which might have limited external validity for a general geriatric population. However, there was no absolute MMSE cut-off below which older persons could not participate, and a considerable proportion (26\%) had a suboptimal MMSE sum score of 24 to 28. Furthermore, the a priori cumulative probability of $7 \%$ for developing dementia during 6 to 8 years of follow-up in our study population was within the range of commonly reported incidence rates among persons of this age range. ${ }^{20}$

\section{Conclusions}

A recent meta-analysis on prediction tools for dementia risk showed that most models have a poor performance in discriminating high-risk from low-risk individuals. ${ }^{21}$ Our present results show that assessing SMC in combination with the MMSE delayed recall item and the VAT appears to be a promising way to assess dementia

\section{Figure 2. Kaplan-Meier curves for dichotomized MMSE-5 and VAT scores, for participants with and without SMC.}

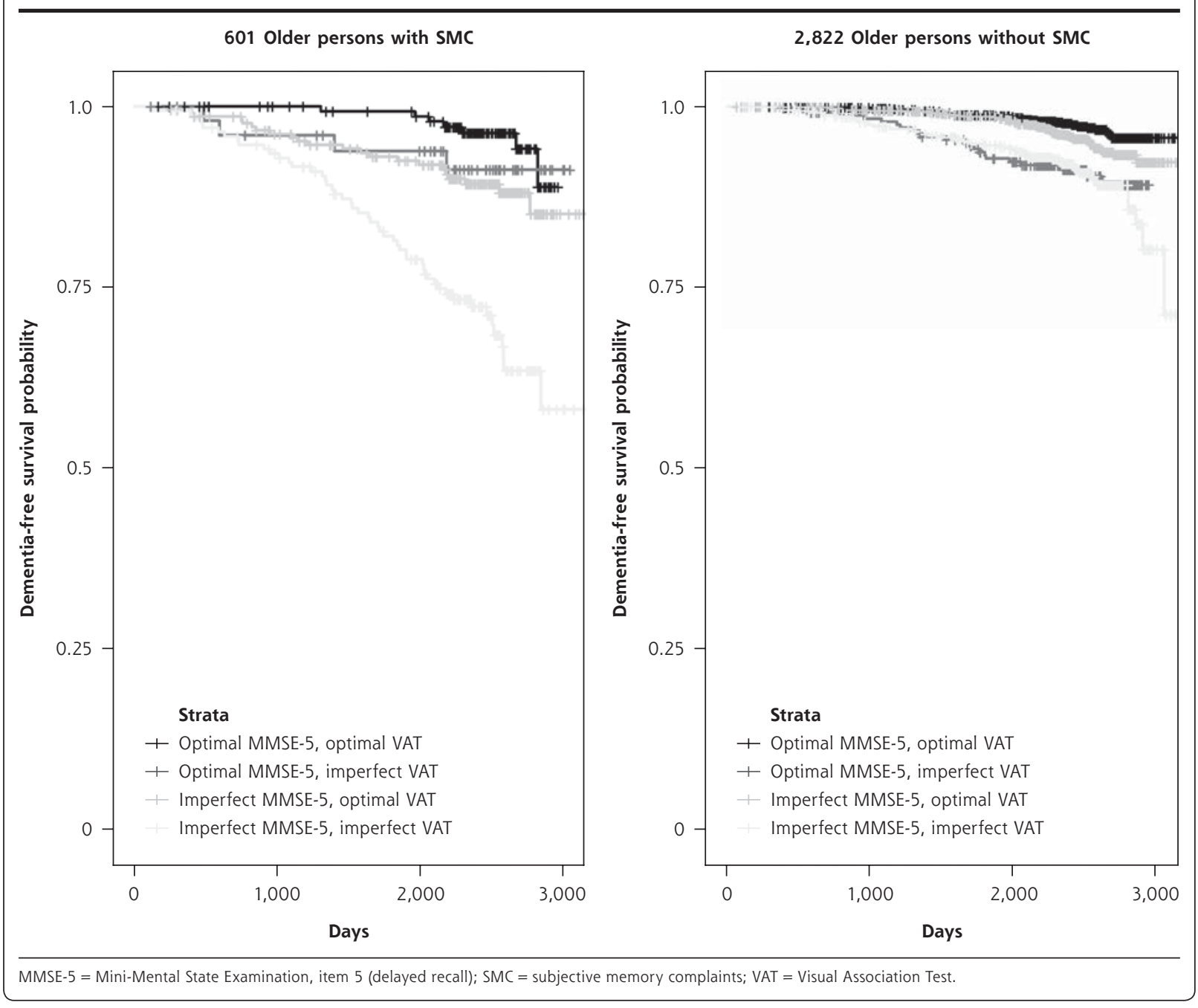


risk in primary care. Because we studied associations instead of risk predictions for dementia, a formal comparison with the models of Stephan et $\mathrm{a}^{21}$ is beyond the scope of the present study. Further study using similar methods is necessary to allow for such a comparison. In conclusion, in participants with SMC, the strength of the association between future dementia and an imperfect MMSE-5 score depended substantially on the VAT score.

To read or post commentaries in response to this article, see it online at http://www.AnnFamMed.org/content/17/5/412.

Submitted September 6, 2018; submitted, revised, March 11, 2019; accepted April 10, 2019.

Key words: dementia; neuropsychological tests; memory; cognition; association learning; self report; geriatric assessment; risk assessment; primary health care

Author contributions: E.P.MvC., E.R., and W.A.vG. conceived and designed the trial; W.A.vG. coordinated the trial; L.L.vW., E.R., S.J., E.P.MvC, and W.A.vG. interpreted the results; L.L.vW. conducted statistical analyses and drafted the manuscript; W.A.vG., E.R., and E.P.MvC. obtained funding. L.L.vW., E.R., S.J., E.P.MvC., and W.A.vG. revised the manuscript for important intellectual content. W.A.vG. is the principal investigator. The authors assume full responsibility for analyses and interpretation of these data.

Funding support: The preDIVA trial was supported by the Dutch Ministry of Health, Welfare and Sport (grant no. 50-50110-98-020), the Dutch Innovation Fund of Collaborative Health Insurances (grant no. 05-234), and the Netherlands Organisation for Health Research and Development (grant no. 62000015). The funders had no role in the study design, data collection and analysis, decision to publish, or preparation of the manuscript.

Trial registration: The preDIVA trial is registered with ISRCTN (no. ISRCTN 29711771).

Acknowledgments: The authors thank the participants of the preDIVA study and everyone involved in its conduction. Specifically, we thank Jan-Willem van Dalen for statistical advice.

Supplemental materials: Available at http://www.AnnFamMed. org/content/17/5/412/suppl/DC1/.

\section{References}

1. de Vugt ME, Verhey FR. The impact of early dementia diagnosis and intervention on informal caregivers. Prog Neurobiol. 2013;110:54-62.

2. Mitchell AJ, Beaumont $H$, Ferguson D, Yadegarfar M, Stubbs B. Risk of dementia and mild cognitive impairment in older people with subjective memory complaints: meta-analysis. Acta Psychiatr Scand. 2014;130(6):439-451.

3. Galasko D, Klauber MR, Hofstetter CR, Salmon DP, Lasker B, Thal LJ. The Mini-Mental State Examination in the early diagnosis of Alzheimer's disease. Arch Neurol. 1990;47(1):49-52.
4. Small BJ, Viitanen M, Backman L. Mini-Mental State Examination item scores as predictors of Alzheimer's disease: incidence data from the Kungsholmen Project, Stockholm. J Gerontol A. 1997;52(5): M299-M304.

5. Ashford JW, Kolm P, Colliver JA, Bekian C, Hsu LN. Alzheimer patient evaluation and the mini-mental state: item characteristic curve analysis. J Gerontol. 1989;44(5):P139-P146.

6. Fuchs A, Wiese B, Altiner A, Wollny A, Pentzek M. Cued recall and other cognitive tasks to facilitate dementia recognition in primary care. J Am Geriatr Soc. 2012;60(1):130-135.

7. Lindeboom J, Schmand B, Tulner L, Walstra G, Jonker C. Visual association test to detect early dementia of the Alzheimer type. J Neurol Neurosurg Psychiatry. 2002;73(2):126-133.

8. Spaan PE, Raaijmakers JG, Jonker C. Early assessment of dementia: the contribution of different memory components. Neuropsychology. 2005;19(5):629-640.

9. Jongstra S, van Gool WA, Moll van Charante EP, et al. Improving prediction of dementia in primary care. Ann Fam Med. 2018;16(3): 206-210.

10. Moll van Charante EP, Richard E, Eurelings LS, et al. Effectiveness of a 6 -year multidomain vascular care intervention to prevent dementia (preDIVA): a cluster-randomised controlled trial. Lancet. 2016; 388(10046):797-805.

11. Richard E, Van den Heuvel E, Moll van Charante EP, et al. Prevention of dementia by intensive vascular care (PreDIVA): a cluster-randomized trial in progress. Alz Dis Assoc Disord. 2009;23(3):198-204.

12. American Psychiatric Association. Diagnostic and Statistical Manual of Mental Disorders. 4th ed. Washington DC: American Psychiatric Association; 1994.

13. Sheikh J, Yesavage J. Geriatric Depression Scale (GDS): recent evidence and development of a shorter version. Clin Gerontol. 1986; 5(1-2):165-173.

14. Folstein MF, Folstein SE, McHugh PR. "Mini-mental state". A practical method for grading the cognitive state of patients for the clinician. J Psychiatr Res. 1975;12(3):189-198.

15. Folstein MF, Folstein SE, McHugh PR. "Mini-mental state". A practical method for grading the cognitive state of patients for the clinician. Kok R, Verhey F, Dutch trans; 2002.

16. RStudio Team. RStudio: Integrated Development for R. Boston, MA: RStudio Inc; 2016. http://www.rstudio.com/.

17. Therneau TM, Lumley T. A Package for Survival Analysis in S. Version 2.38; 2015. https://CRAN.R-project.org/package = survival.

18. Kassambara A, Kosinski M, Biecek P, Fabian S. Survminer: Drawing Survival Curves Using 'ggplot2'. R Package version 0.4.2; 2018. https:// CRAN.R-project.org/package $=$ survminer.

19. Kaup AR, Nettiksimmons J, LeBlanc ES, Yaffe K. Memory complaints and risk of cognitive impairment after nearly 2 decades among older women. Neurology. 2015;85(21):1852-1858.

20. van Bussel EF, Richard E, Arts DL, et al. Dementia incidence trend over 1992-2014 in the Netherlands: analysis of primary care data. PLoS Med. 2017;14(3):e1002235.

21. Stephan BC, Kurth T, Matthews FE, Brayne C, Dufouil C. Dementia risk prediction in the population: are screening models accurate? Nature Rev Neurol. 2010;6(6):318-326. 